\title{
Study of Gravitational Lensing Constraints on the Cardassian Model Based on the Friedmann Equation
}

\author{
Bani Mukherjee (Corresponding author) \& Bijay Kumar Mandal \\ Department of Applied Mathematics, Indian School of Mines University \\ Dhanbad-826004, Jharkhand, INDIA
}

Tel: 91-943-112-5407Ｅ-mail: mukherjeebani@gmail.com

\begin{abstract}
The Cardassian Model for the dark energy, dark matter and unified theory, which have usually invoked as the most plausible way to explain the recent observational result, have been studied. In this paper we mainly focus our attention to investigate some observational consequences of a flat, matter dominated and accelerating scenario, on the constraints, the parameters $n$ and $q$ which fully characterize the Cardassian Model. The dependence of the acceleration red-shift that is the red-shift at which the Universe begins to accelerate, with the parameters $n$ and $q$ is briefly discussed. When we consider $q=1$ our case 2.1 converges to the case of Cardassian model for the dark energy by S. Sen ${ }^{44}$. Moreover when we consider $\Omega_{r 0}$, the density parameter for radiation, is zero then our case 2.1 converges to the case of Cardassian model for dark matter by A., Dev ${ }^{20}$, as a special case.
\end{abstract}

PACS : 98.80 , Es : $95.35+d ; 98.62 \mathrm{Sb}$

Keywords: Cardassian model, Dark matter, Dark energy, Accelerating universe

\section{Introduction}

The current observations indicate that we are living in as spatially flat, low matter density Universe which is currently undergoing an accelerating expansion (P. De, Bernadis ${ }^{11}$; S. Hanany ${ }^{26}$; A. Balbi ${ }^{3}$; S. Perlmutter ${ }^{34,35}$; P.M. Garnavich ${ }^{23}$; A. G. Rieses ${ }^{37}$ ). The most simple explanation of the current cosmological state of the universe requires two dark components: one is in the form of non-relativistic dust (dark matter) with vanishing pressure contributing one-third of the total energy density of the universe and clustering gravitationally at small scales while the second one is a smoothly distributed component having large negative pressure (dark energy) and contributing around two-third of the total energy density of the universe.

As none of the two components (dark matter and dark energy) has laboratory evidence both directly or indirectly, one have to invoke untested physics twice to explain the current observations. That is why people in recent times have proposed interesting scenarios where one describes both dark matter and dark energy in a unified way through a single fluid component in the Einsteins equation. Chaplygin gas model is one such interesting possibility which has attracted lot of attentions in recent times (A. Kamenshchik ${ }^{28}$; M.C. Bento ${ }^{8,9}$; N. Bilic ${ }^{13}$ ). Padmanabhan and Roy Choudhury have also proposed an interesting unified description based on a rolling tachyon arising in string theory (T. Padmanabhan ${ }^{32}$ ).

Although the simplest candidate for this dark energy is the vacuum energy or the cosmological constant $(\Lambda)$, alternative scenarios where the acceleration is driven by dynamical scalar field both minimally (R.R. Caldwell ${ }^{15}$; P.J.E. Peebles ${ }^{33}$; P. G. Ferreira ${ }^{21}$; E. J. Copeland ${ }^{18}$; P.J. Steinhardt ${ }^{46}$; I. Zlatev ${ }^{54}$; C. Wetterich ${ }^{51}$; B. Ratra ${ }^{36}$; T. Barreirs ${ }^{4}$; V. Sahni ${ }^{38}$; A. A. $\mathrm{Sen}^{42}$; M. C. Bento ${ }^{7}$ ) and non-minimally (N. Berlolo ${ }^{10}$; O. Bertolami ${ }^{12}$; J. P. Uzan ${ }^{49}$; L. Amendola ${ }^{1,2}$; M. Gasperini ${ }^{24,25}$; A. A. Sen ${ }^{40,41,42}$ ) coupled with gravity called quintessence have been widely investigated in recent years $\left(\mathrm{N}\right.$. Benerjee ${ }^{5,6}$; S. Sen ${ }^{45}$; T. $\left.\mathrm{Chiba}^{16}\right)$.

In particular, Y. Wang ${ }^{50}$ has studied some observational characteristics of a direct generalization of the original Cardassian model. According to these authors, the observational expressions in this new scenarios are very different from generic quintessence cosmologies and fully determined by two dimensionless parameters $\mathrm{n}$ and q. They proposed the interesting alternative to quintessence scenario where the recent acceleration of the flat universe is driven solely by the matter, instead of using any cosmological constant or vacuum energy term. Since pure matter or radiation cannot alone take into account the recent acceleration in the flat universe, this goal is accomplished by modifying the Friedman equation with 
the empirical additional term named Cardassian term

$$
H^{2}=A \rho+B \rho^{n}
$$

where $A=8 \pi G / 3, B$ and $n$ are constants and are the parameters of the model. Here the energy density $(\rho)$ contains only matter $\left(\rho_{m}\right)$ and radiation $\left(\rho_{r}\right)$ i.e. $\rho=\rho_{m}+\rho_{r}$. Since at present $\rho_{m}>>\rho_{r}, \rho$ can be considered consisting of $\rho_{m}$ only.

Observational constraints from a variety of astronomical data have been also investigated recently, both in the original Cardassian model (Z. H. Zhu ${ }^{53}$; S. Sen ${ }^{44}$; A.A. Sen ${ }^{43}$ ) and its generalized versions (T. Multamaki ${ }^{31}$ ). Although J. M. Cline $^{17}$ has shown that cardassian model based on this higher dimensional interpretation, violates the weak energy condition for the bulk stress energy for $n<2 / 3$ which is necessary for accelerating universe in late times. This extra term may also arise due to the matter self interactions that contributes a negative pressure, through a long-range confining force which may be of gravitational origin.

The aim of this paper is to explain some observational constraints on the Generalized Cardassian (GC) \& Modified Polytropic Cardassian (MPC) Model for the dark energy \& dark matter scenarios. We mainly focus our study on the constraints that is the free parameters of the model ( $n$ and $q)$.

\section{The Freidmann Model :}

In Standard cosmology, the evolution of the universe, is governed by the Friedmann equation

$$
H^{2}=\frac{8 \pi G_{\rho}}{3}
$$

At the current epoch the critical density is

$$
\rho_{\text {crit }}=\frac{3 H_{0}^{2}}{8 \pi G}=(1.054)\left(10^{-5}\right) h^{2} \mathrm{Gev} / \mathrm{cm}^{3}
$$

where subscript refers to the present day.

$$
H_{0}=100 h \mathrm{Km} / \mathrm{s} / \mathrm{Mpc}
$$

The ratio of energy density to the critical density

$$
\Omega=\rho / \rho_{\text {crit }},
$$

In the standard picture, an additional component beyond matter and radiation is assumed to reach the critical density. This component is taken to be a vacuum energy; a cosmological constant $\Lambda$ or a time dependent vacuum energy or scalar field known as quintessence that evolves dynamically with time.

The Hubble parameter can be related to its present day value by:

$$
H=H_{0} E_{F}(z)
$$

where

$$
E_{F}(z)^{2}=\Omega_{r 0}(1+z)^{4}+\Omega_{m 0}(1+z)^{3}+\Omega_{x 0}(1+z)^{3(1+w x)}
$$

where the current contributions from radiation $\left(=\Omega_{r 0}\right)$, matter $\left(=\Omega_{m 0}\right)$ and vacuum $\left(=\Omega_{x 0}\right)$ with $w x=(p x / \rho x)$, the equation of state.

\section{Formulation of the model}

\section{Case1: Generalized Cardassian (GC) model based on The Friedmann equation}

In the Cardassian model (K. Freese ${ }^{22}$ ) the Friedmann equation has the general form

$$
H^{2}=g\left(\rho_{M}\right),
$$

where the $\rho_{M}$ is the energy density of ordinary matter and radiation. The Universe is assumed to be flat and there is neither new type of matter nor a non-zero cosmological constant. The function $g$ is assumed to approach the standard form, $k^{2} \rho$ at early times, to give accelerated expansion in accordance with the supernova observation. Since the behaviour of the function is different at different values of $\rho_{M}$, there is an associated scale, $\rho_{c}$, or red-shift $Z_{\text {eq }}$, in the function $g$ that determines when the evolution is standard and when the non-standard terms begin to dominate.

The original Cardassian form of $g(\rho)$ (omitting the subscript $M$ ) is

$$
H^{2}=A \rho+B \rho^{n}, \text { with } n<2 / 3
$$

At early times the universe is dominated by the $A \rho$ term, provided that $B$ is small enough at the time of interest. At late times the $\rho^{n}$ term becomes significant, providing acceleration compared to the standard case. In terms of the scale $\rho_{c}$, equation (3) can be written as

$$
H^{2}=A \rho\left[1+\left(\rho / \rho_{c}\right)^{n-1}\right]
$$


Hence $B=A\left(\rho_{c}\right)^{1-n}$.

In a matter dominated universe this is conveniently parameterized by the red-shift at which the two terms are equal, $Z_{e q}$ (or $Z_{\text {card }}$ )

$$
H^{2}=A \rho\left[1+\left(1+Z_{e q}\right)^{3(1-n)}\right],
$$

\section{Case 2: Modified Polytropic Cardassian (MPC) model based on the Friedmann equation}

Recently another Cardassian model has been studied by $Y$. Wang ${ }^{50}$. In this Modified Polytropic Cardassian (MPC) model, the Friedmann equation is given by

$$
H^{2}=A \rho\left[1+\left(\rho / \rho_{\text {card }}\right)^{q(n-1)}\right]^{1 / q},
$$

where $\rho_{\text {card }}$ (or $\rho_{c}$ ) is again the energy density of matter at which the non-standard terms begin to dominate and $q>0$ is deceleration parameter. The MPC model is constrained by the supernova observations as well as the CMB The growth of gravitational instabilities in the Modified Polytropic Cardassian model described by equation (6). The original Cardassian model is a special case of the MPC model with $q=1$.

Case 2.1 : Once the energy density $\rho$ drops below $\rho_{\text {card }}$ the universe starts accelerating, following S. Sen ${ }^{44}, \rho_{\text {card }}$ has been rewrite as follow :

$$
\rho_{\text {card }}=\rho_{m 0}\left(1+Z_{\text {card }}\right)^{3}\left\{1+\frac{\Omega_{r 0}}{\Omega_{m 0}}\left(1+Z_{\text {card }}\right)\right\}
$$

where $\rho_{\text {card }}$ (or $\rho_{c}$ ) is the energy density and $Z_{\text {card }}$ is the red shift at which the second term in equation (1), starts dominating over the first term. The MPC Model have the three parameter, one $B$ (or $\rho_{\text {card }}$ or $Z_{\text {card }}$ ), second $q$ (or deceleration parameter) and third $n$ (power law index parameter). The current contributions from radiation $\left(=\Omega_{r 0}\right)$ and matter $\left(=\Omega_{m 0}\right)$, the two parameters are defined as $\Omega m 0=\frac{\rho_{m 0}}{\rho_{c r i t}}$ and $\Omega r 0=\frac{\rho_{r 0}}{\rho_{c r i t}}$ respectively.

Substituting the value of $\rho_{\text {card }}$ from equation (13), equation (12) at current contributions becomes

$$
H_{0}^{2}=A \rho_{0}\left[1+\left(\frac{\rho_{0}}{\rho_{m 0}}\right)^{q(n-1)}\left(1+Z_{c a r d}\right)^{3 q(1-n)}\left\{1+\frac{\Omega_{r 0}}{\Omega_{m 0}}\left(1+Z_{c a r d}\right)\right\}^{q(1-n)}\right]^{\frac{1}{q}}
$$

or,

$$
H_{0}^{2}=A \rho_{0}\left[1+\left(\frac{\rho_{0}+\rho_{m 0}}{\rho_{m 0}}\right)^{q(n-1)}\left(1+Z_{c a r d}\right)^{3 q(1-n)}\left\{1+\frac{\Omega_{r 0}}{\Omega_{m 0}}\left(1+Z_{c a r d}\right)\right\}^{q(1-n)}\right]^{\frac{1}{q}}
$$

or,

$$
H_{0}^{2}=\frac{8 \pi G}{3} \rho_{0}\left[1+\left(1+\frac{\Omega_{r 0}}{\Omega_{m 0}}\right)^{q(n-1)}\left(1+Z_{c a r d}\right)^{3 q(1-n)}\left\{1+\frac{\Omega_{r 0}}{\Omega_{m 0}}\left(1+Z_{\text {card }}\right)\right\}^{q(1-n)}\right]^{\frac{1}{q}}
$$

or,

$$
\frac{3 H_{0}^{2}}{8 \pi G \rho_{0}}=\left[1+\left(1+\frac{\Omega_{r 0}}{\Omega_{m 0}}\right)^{q(n-1)}\left(1+Z_{c a r d}\right)^{3 q(1-n)}\left\{1+\frac{\Omega_{r 0}}{\Omega_{m 0}}\left(1+Z_{c a r d}\right)\right\}^{q(1-n)}\right]^{\frac{1}{q}}
$$

or, from equation (3) considering $\rho_{\text {crit }}=\frac{3 H_{0}^{2}}{8 \pi G}$ we have

$$
\frac{\rho_{\text {crit }}}{\rho_{0}}=\left[1+\left(1+\frac{\Omega_{r 0}}{\Omega_{m 0}}\right)^{q(n-1)}\left(1+Z_{\text {card }}\right)^{3 q(1-n)}\left\{1+\frac{\Omega_{r 0}}{\Omega_{m 0}}\left(1+Z_{\text {card }}\right)\right\}^{q(1-n)}\right]^{\frac{1}{q}}
$$

or,

$$
\frac{\rho_{0}}{\rho_{\text {crit }}}=\left[1+\left(1+\frac{\Omega_{r 0}}{\Omega_{m 0}}\right)^{q(n-1)}\left(1+Z_{\text {card }}\right)^{3 q(1-n)}\left\{1+\frac{\Omega_{r 0}}{\Omega_{m 0}}\left(1+Z_{\text {card }}\right)\right\}^{q(1-n)}\right]^{\frac{-1}{q}}
$$

or,

$$
\frac{\rho_{0}}{\rho_{\text {crit }}}=\frac{\rho_{m 0}+\rho_{r 0}}{\rho_{\text {crit }}}=\Omega_{m 0}+\Omega_{r 0}=F
$$

Where

$$
F=\left[1+\left(1+\frac{\Omega_{r 0}}{\Omega_{m 0}}\right)^{q(n-1)}\left(1+Z_{\text {card }}\right)^{3 q(1-n)}\left\{1+\frac{\Omega_{r 0}}{\Omega_{m 0}}\left(1+Z_{\text {card }}\right)\right\}^{q(1-n)}\right]^{\frac{-1}{q}}
$$

The Original Cardassian model is a special case of the MPC model with $q=1$. When we consider $q=1$ our MPC model case converge to the original Cardassian model case of S. Sen ${ }^{44}$.

$$
F=\left[1+\left(1+\frac{\Omega_{r 0}}{\Omega_{m 0}}\right)^{(n-1)}\left(1+Z_{c a r d}\right)^{3(1-n)}\left\{1+\frac{\Omega_{r 0}}{\Omega_{m 0}}\left(1+Z_{\text {card }}\right)\right\}^{(1-n)}\right]^{-1}
$$


Observational constraints from a variety of astronomical data have been also investigated recently, both in the original Cardassian model (Z.H. Zhu ${ }^{53}$; S. Sen ${ }^{44}$; A. A. Sen ${ }^{43}$ ) and in its generalized versions (T. Multamaki ${ }^{31}$ ). Perhaps the most interesting feature of these models is that although being matter dominated, they may be accelerating and can still reconcile the indications for a flat universe $\left(\Omega_{\text {total }}=1\right)$ from CMB observations with the clustering estimates that point consistently to $\Omega_{m} \simeq 0.3$ with no need to invoke either a new dark component or a curvature term. In these scenarios, it happens through a redefinition of the value of the critical density (Z. H. Zhu ${ }^{53}$; J. M. Cline ${ }^{17}$ ). Hence equation (15) becomes

$$
F=\left[1+\left(1+Z_{\text {card }}\right)^{3 q(1-n)}\right]^{-\frac{1}{q}}
$$

which converges to the case of Cardassian model for dark matter by A. $\operatorname{Dev}^{20}$, as a special case.

\section{Lensing constraints}

In this section we use statistics of gravitationally lensed quasars to place limits on the free parameters of GC scenarios. We work with a sample of $867(z>1)$ high luminosity optical quasars. Our sample consists of data from the following optical lens surveys: HST Snapshot survey (D. Maoz ${ }^{30}$ ), Crampton survey (D. Crampton ${ }^{19}$ ), Yee survey (H. K. C. Yee ${ }^{52}$ ), Surdej survey (S. Surdej ${ }^{47}$ ), NOT Survey (A.O. Jaunsen ${ }^{27}$ ) and FKS survey (C. S. Kochanek ${ }^{29}$ ). Since the main difference between the analyses performed in this section and the previous ones that use gravitational lensing statistics to constrain cosmological parameters is the cosmological model that here is being considered.

\section{Conclusion}

The possibility of an accelerating from distance measurements of type Ia-supernovae constitutes one of the most important results of modern cosmology. In figure $(1 a, 1 b, 1 c, 1 d)$, we show a generalized version of the figure of equation (15) in which the value of $\Omega_{m 0}=0.05$ and 0.0000989 and plane $Z_{\text {card }} n$ is displayed for selected values of $q$ for certain values of $F(0.1,0.2,0.3,0.4)$. However, gravitational cluster (R. G. Calberg ${ }^{14}$ ) and other data suggest (M.S. Turner ${ }^{48}$ ), the total matter density to be $30 \%$ of the usual critical density i.e., $\rho_{0}=0.3 \rho_{\text {crit }}$. This sets a preferred value 0.3 for $F$.

In figure $(2 a, 2 b, 2 c, 2 d)$, we show the figure of equation (17), in which the plane $Z_{c a r d} n$ is displayed for selected values of $q$ for certain values of $F(0.1,0.2,0.3,0.4)$ and which converges to the case of Cardassian model for dark matter by A. $\operatorname{Dev}^{20}$. as a special case.

Again we draw the figures $(3 a, 3 b, 3 c, 3 d)$ by taking the value of $\Omega_{m 0}=0.05$ and $\left.\Omega_{r 0}=(0.3-0.05)\right)$. we show a generalized version of the figure of equation (15) in which the plane $Z_{\text {card }} n$ is displayed for selected values of $q$ for certain values of $F(0.1,0.2,0.3,0.4)$.

\section{References}

Amendola, L. (1999). Phys. Rev. D, 60, 043501

Amendola, L. (2000). Phys. Rev. D, 62, 043511

Balbi, A. et al. (2000). astir-ph/00051245.

Barreiro, T., Copeland, E.J. and Nunes, N.J. (2001). Phys. Rev. D, 61, 12730

Benerjee, N. And Pavon, D. (2001). Phys. Rev. D., 63, 043504.

Benerjee, N. And Pavon, D. (2001). Class. Quant. Grav, 18, 593.

Bento, M. C., Bartolami, O. and Santos, N.C. (2001). astro-ph/0106405.

Bento, M. C.,Bartolami, O. and Sen, A. A. (2002a). Phys. Rev. D, D66, 043507.

Bento, M. C., Bartolami, O. and Sen, A. A. (2002b). astro- ph/ 0210468

Berlolo, N. and Peitroni, M. (1999). Phys. Rev. D., 61, 023518.

Bernardis, P.De et al. (2000). Nature 404, 955

Bertolami, O. and Martins, P. J. (2000). Phys. Rev. D, 61, 064007.

Billic, N., Yupper, G.B. and Viollier, R.D. (2002). Phys. Lett. B, 535, 17

Calberg, R.G. et al. (1996). ApJ. 462, 32.

Caldwell, R. R., Dav. R., and Steinhalt, P. J. (1998). Phys. Rev. Lett., 80, 1582

Chiba, T. (1999). Phys. Rev. D, 60, 083508.

Cline, J.M. and Vinet, J. (2002). hep-ph/ 0211284.

Copeland, E. J., Liddle, A. R. And Wands, D. (1988). Rev. D. 57, 123504

Crampton, D., McClure, R. And Fletcher, M. (1992). astro-phs. J. 392, 23 
Dev, A., Alcaniz, J.S. and Jain, D. (2003). astro-phys/0305068.

Ferreira, P.G. and Joyee, M. (1987). Phys. Rev. Lett., 79, 4740.

Freese, K. and Lewis, M. (2002). Phys. Lett B, 540, 1.

Garnavich, P. M. Et al. (1998). ApJ, 493, L53.

Gasperini, M. (2001a). gr-qc/01050821.

Gasperini, M., Piazza, F. and Veneziano, G. (2001b). gr-qc/ 0108016.

Hanany, S. et al. (2000). astro-ph/0005123.

Jaunsen, A. O., Jablonski, M., Petterson, B. R. and Stabell, R. (1995). Astron. astro-ph. 300, 323.

Kamenshchik, A., Moschella, U., and Pasquier, V. (2001). Phys. Lett, B. 511, 265

Kochanek, C. S., Falco, E. E. Schild, R. (1995). astro-phs. 452, 109.

Maoz, D. et. al. (1993). astro-phs. J., 409, 28

Multamaki, T., Gaztanaga, E., and Manera, M. Submitted to MNRAS. Astro-ph/0303526

Padmanabhan, T. And Roy Choudhury, T. (2002). Phys. Rev. D, 66, 081301(R)

Peebles, P.J.E and Ratra, B. (1988). Apj, 325, L17.

Perlmutter, S. et al. (1997). ApJ, 483, 565.

Perlmutter, S. et al. (1998). Nature, 391, 51.

Ratra, B. and Peebles, P.J.E. (1988). Phys. Rev. D, 37, 3406.

Riess, A. G. (1998). AJ, 116, 1009.

Sahni, V. and Wang, L. (2000). Phys. Rev. D, 37, 3406

Sen, A. A., Sen and Sethi, S. (2001). Phys. Rev. D 63, 107501.

Sen, A. A. and Sen, S. (2001a). Phys. Rev. D, 63, 124006.

Sen, A. A. and Sen, S. (2001b). Mod. Phys. Lett A, 16, 1303

Sen, A. A. and Sethi, S. (2002). Phys. Lett. B, 532, 159.

Sen., A. A. and Sen, S. (2003). astro-ph/0303383

Sen, S. and Sen, A. A. (2003). astro-ph /0211634;

Sen, S. and Seshadri, T. R. (2000). gr-qc /0007079.

Steinhardt, P.J., Wang, L, and Zlatev, I. (1999). Phys. Rev. Lett., 59, 123504

Surdej, J. et. al. (1993). Astron. J. 105, 2064.

Turner, M.S. (2001). astro-ph/0106035.

Uzan, J. P. (1999). Phys Rev. D, 59, 123510.

Wang Y., Freese K., Gonodolo P., Lewis M . (2003). ( astro-ph/0302064)

Wetterich, C. (1988, Nuclear Phys. B, 902668.

Yee, H. K. C., Filippenko and Tang, D. (1993). Astronomy J. 105, 7

Zhu, Z. H., Fuzimoto, M. K., Astrophys. J., 581, 1 (2002). Astrophys J. 585, 52(2003);

Zlatev, I. Wang, L. and Steinhardt, P.J. (1999). Phys. Rev. Lett., 82, 896. 

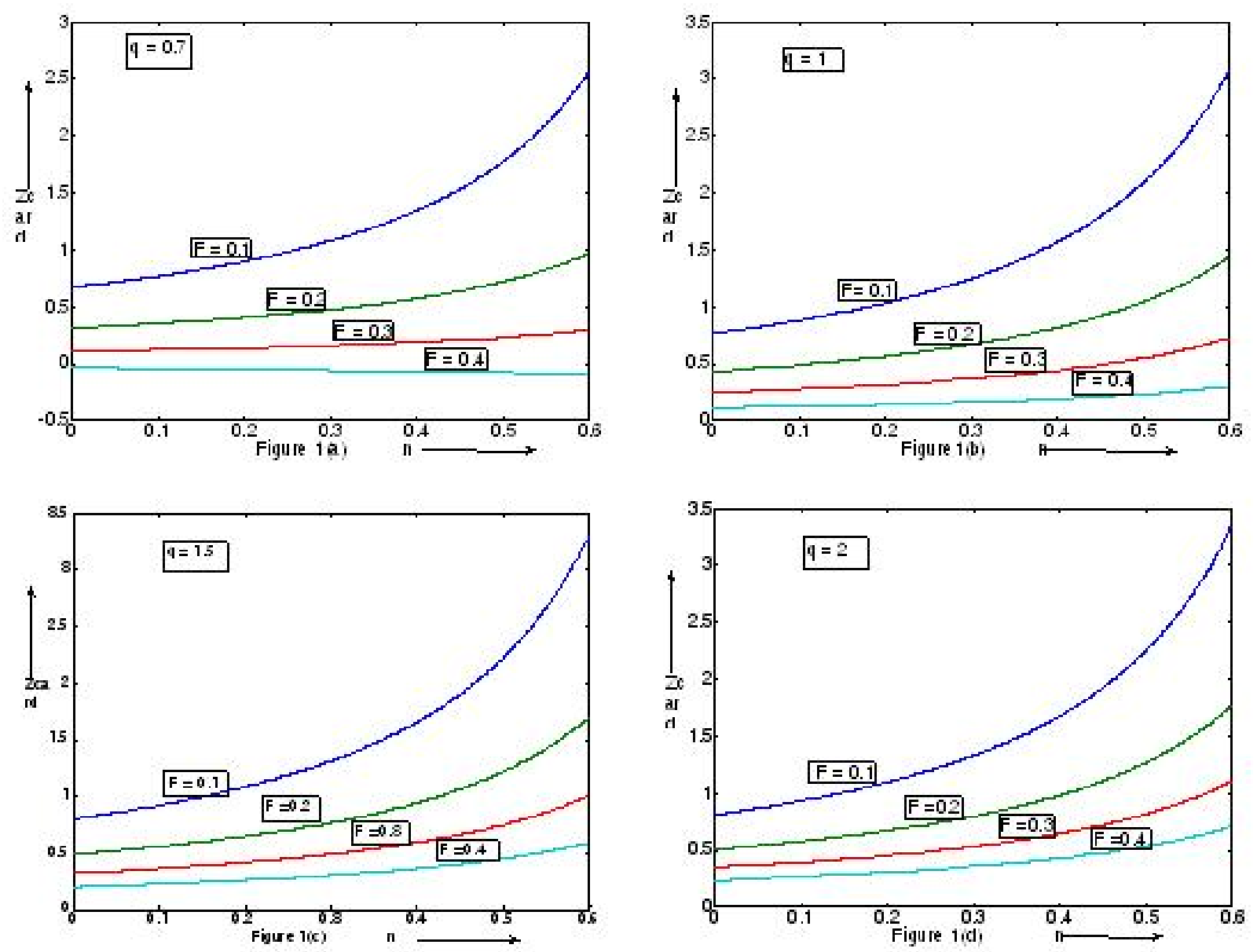

The figure 1(b) converge the case of S. Sen ${ }^{44}$. 

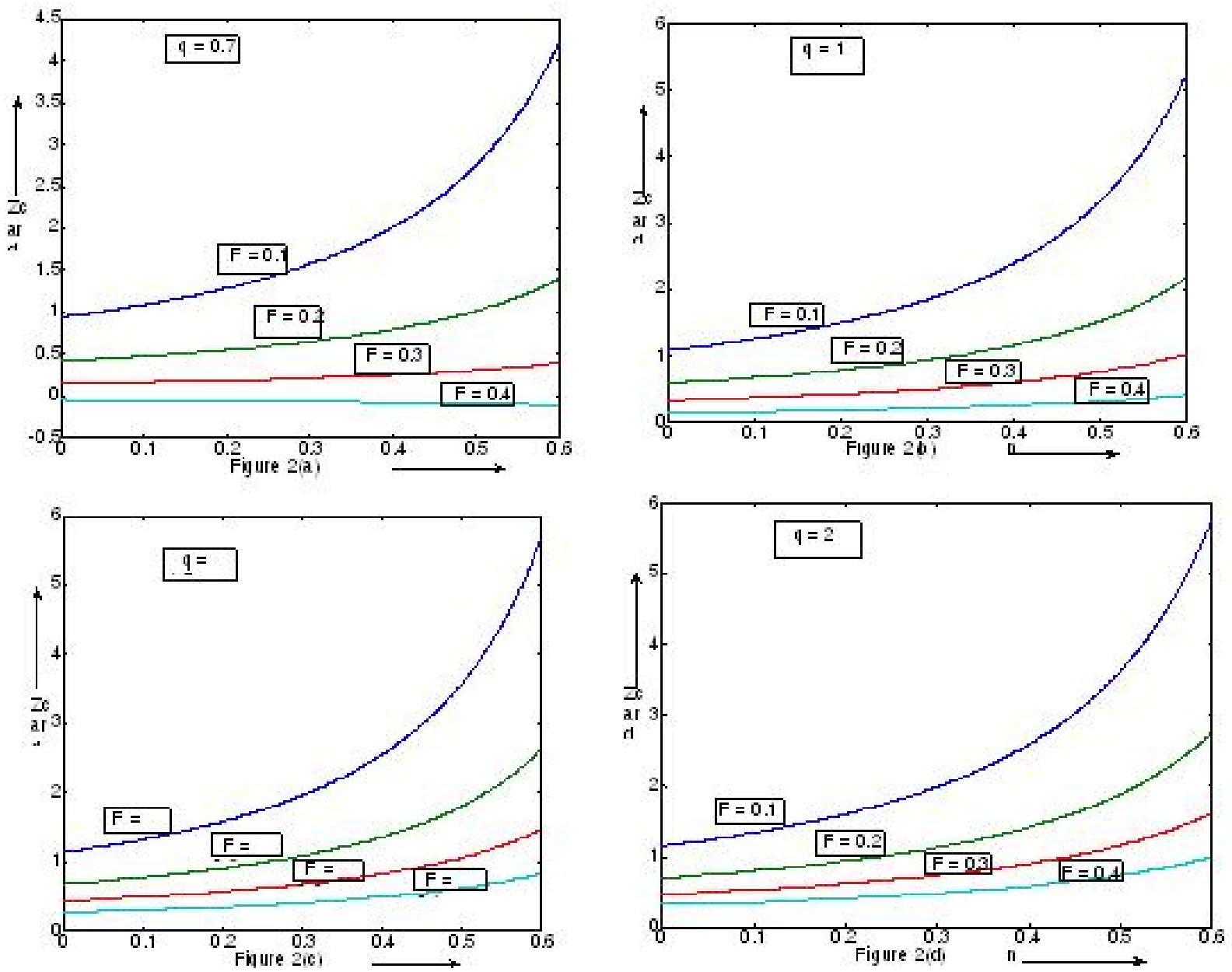

$Z_{\text {card }}$ - $n$ diagram for certain values of $F=\frac{\rho_{0}}{\rho_{\text {crit }}}$ using equation (17) for selected values of $q$. The contours are labelled indicating the corresponding fractin of the standard critical density. 

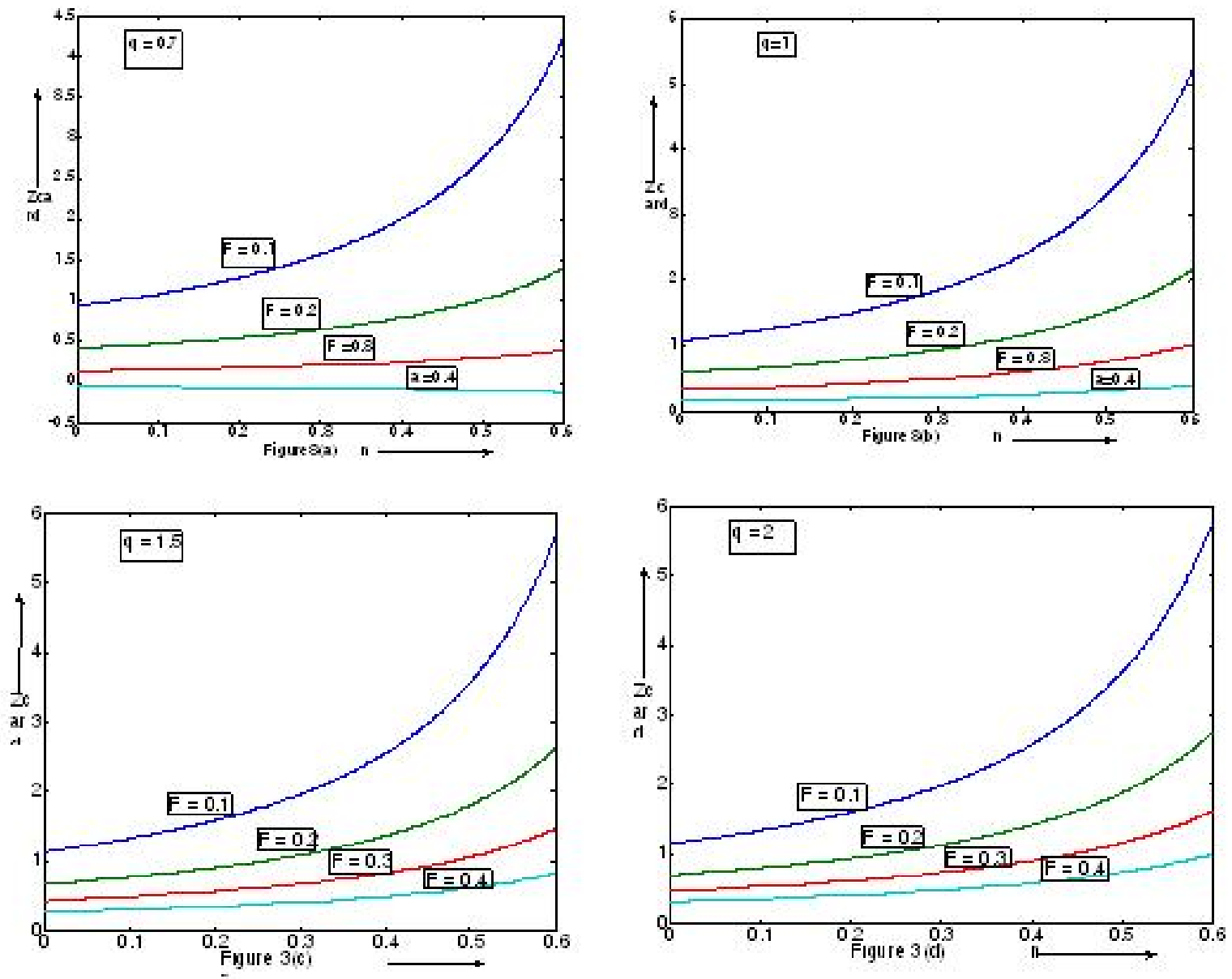

The figure 3(b) also converges to the case of S. Sen ${ }^{44}$. 